\title{
Anatomical resection versus non-anatomical resection for hepatocellular carcinoma: A propensity score matched analysis
}

\author{
Jae Hyun KWON' ${ }^{1}$ Jung-Woo LEE*1, Jong Woo LEE', Young-Joo LEE ${ }^{2}$
}

'Department of Surgery, Hallym University Sacred Heart Hospital, Hallym University College of Medicine, Anyang, Korea

${ }^{2}$ Division of Hepato-Biliary-Pancreatic Surgery, Department of Surgery, Asan Medical Center, University of Ulsan College of Medicine, Seoul, Korea

Introduction: The relative benefit of anatomic resection (AR) versus non-anatomic resection (NAR) of hepatocellular carcinoma (HCC) remains controversial. The objective of this study is to review and compare survival outcomes and recurrence rates of HCC according to tumor size and extent of resection.

Methods: Data of patients with HCC who underwent curative surgical resection between January 1999 and December 2009 from Asan Medical Center were retrospectively reviewed. To compare survival outcomes between AR and NAR, propensity score matching (PSM) was conducted according to tumor size. A total of 986 patients were analyzed; 812 patients received AR and 174 patients underwent NAR.

Results: The 5-year OS rate of HCC less than $5 \mathrm{~cm}$ was $78.1 \%$ and $62.4 \%$ in AR and NAR, respectively $(p=0.002)$. The 5 -year RFS rate of HCC less than $5 \mathrm{~cm}$ was $48.2 \%$ and $36.8 \%$ in AR and NAR, respectively ( $p=0.017$ ). The 5 -year OS rate of HCC greater than or equal to $5 \mathrm{~cm}$ was $48.6 \%$ and $47.1 \%$ in AR and NAR, respectively $(p=0.629)$. The 5 -year RFS rate of HCC greater than or equal to $5 \mathrm{~cm}$ was $30.4 \%$ and $27.5 \%$ in AR and NAR, respectively $(p=0.462)$. After PSM, the OS and RFS rate of HCC less than $5 \mathrm{~cm}$ were significantly better in the AR group. However, in HCC greater than or equal to $5 \mathrm{~cm}$, there were no significant difference between the AR and the NAR group.

Conclusions: AR in HCC less than $5 \mathrm{~cm}$ decreased the risk of tumor recurrence and improved OS. In HCC with diameter over $5 \mathrm{~cm}$, AR and NAR showed comparable survival outcomes. 